\title{
An efficient installation for applets to be post-issuance of Java Card system using an IC chip
}

\author{
Won-Ho Choi ${ }^{\text {a)}}$, Seung-Joon Seok, and Min-Soo Jung \\ Department of Computer Engineering, University of Kyungnam, \\ 449 Wolyung-Dong, Chnagwon, Kyungnam 631-701, Korea \\ a)hoya9499@kyungnam.ac.kr
}

\begin{abstract}
Most smart cards based on the Java Card need an installer for deploying a new applet into its memory after the cards were issued. Installing the applet takes lots of time because of unique components downloading and hardware limitations. An efficient installation for applets in the Java Card platform is investigated. The proposed scheme could improve applet installation time significantly compared to the conventional scheme by fully utilizing available RAM space and could present the best a block size to be transferred between a terminal and JCRE.
\end{abstract}

Keywords: IC card, Java Card, JCRE, installation, resolution, postissuance

Classification: Integrated circuits

\section{References}

[1] Sun Microsystems, Inc., Virtual Machine Specification, Java Card ${ }^{\mathrm{TM}}$ Platform, Version 2.2.1, Oct. 2003.

[2] Sun Microsystems, Inc., Runtime Environment Specification, Java Card $^{\mathrm{TM}}$ Platform, Version 2.2.1, Oct. 2003.

[3] U. Hansmann, et al., Smart Card Application Development Using Java, Springer, Aug. 2002.

[4] Z. Chen, Java Card Technology for Smart Cards: Architecture and Programmer's guide (Java Series), Addison-Wesley, June 2000.

[5] D. Davis and D. Balaban, "Wake Up and Smell The Java!," Card Technology Magazine, Feb. 2002.

[6] G. Grimaud and J. J. Vandewalle, "Introducing Research Issues for Next Generation Java-based Smart Card Platforms," Proc. Smart Objects Conference, Grenoble, France, pp. 138-141, May 2003.

[7] W. Rankl and W. Effing, Smart Card Handbook, the third Edition, John Wiley \& Sons, Jan. 2004.

[8] M. Baentsch, P. Buhler, P. T. Eirich, F. Hring, and M. Oestreicher, "Java Card - From Hype to Reality," IEEE Concurrency, vol. 7, no. 4, pp. $36-43$, Oct. 2002.

[9] B. Venners, Inside the JAVA Virtual Machine Second Edition, McGrawHill Companies, Jan. 2000. 
[10] Samsung Electronics, User's Manual S3FS9QB 32-Bit CMOS Microcontroller for Smart Card Revision1, Sept. 2005.

\section{Introduction}

Java Card has recently been recognized as a dominating platform for smart cards and SIM/USIM cards using an Integrated Circuit (IC) chip [1, 2, 3, $5,6,8]$. The Java Card system is issued with previously made applets and is also able to download a new applet whenever users or vendors need after it is issued. These methods are called pre-issuance and post-issuance $[1,2$, 3]. In order to download a new applet is different from a standard Java platform because the Java Card platform runs a tiny Java Card Runtime Environment (JCRE) optimized for an embedded device with limited memory and computing power in the IC chip. There are some reasons why the Java Card consumes much time in installing a new applet. First of all, applets and its objects are stored into Flash memory of the JCRE in the IC chip. The cost of RAM for the Java Card is still expensive, so many manufacturers more use Flash as storage. However, writing data to Flash cells is more 1,000 times slow than RAM memory [7]. Second of all, applets to be postissuance need a process of memory mapping. References in the applet code to be developed must be resolved and updated to refer to direct references in the JCRE memory space during the installation process. The reference resolution performed during an applet installation creates significant memory copy overhead incurred by writing data into Flash multiple times.

We have investigated an efficient installation for an applet in the Java Card platform using an IC chip. The major scheme of the installation is to fully utilize available RAM buffer size to significantly reduce memory copy overhead during reference resolution processes. It exploits efficient block sizes for transferring components with reduced acknowledgement APDU messages.

\section{Experimental}

In the conventional Java Card platform, because of limited RAM space, the installer applet in the on-card platform first downloads the target applet CAP file components into Flash and copies them into RAM when reference resolution is performed. In general, because Flash memory writing speed is more than 1,000 times slower than that of RAM, the reference resolution performed during an applet installation creates significant memory copy overhead incurred by writing data into Flash multiple times.

The proposed efficient installation scheme is summarized as like that the scheme fully utilizes available RAM buffer size to significantly reduce memory copy overhead incurred by the redundant Flash copies during reference resolution processes. Our scheme exploits efficient mode for transferring components with reduced acknowledgement APDU messages. Based on the statistical characteristics of an applet being installed, the scheme empirically 


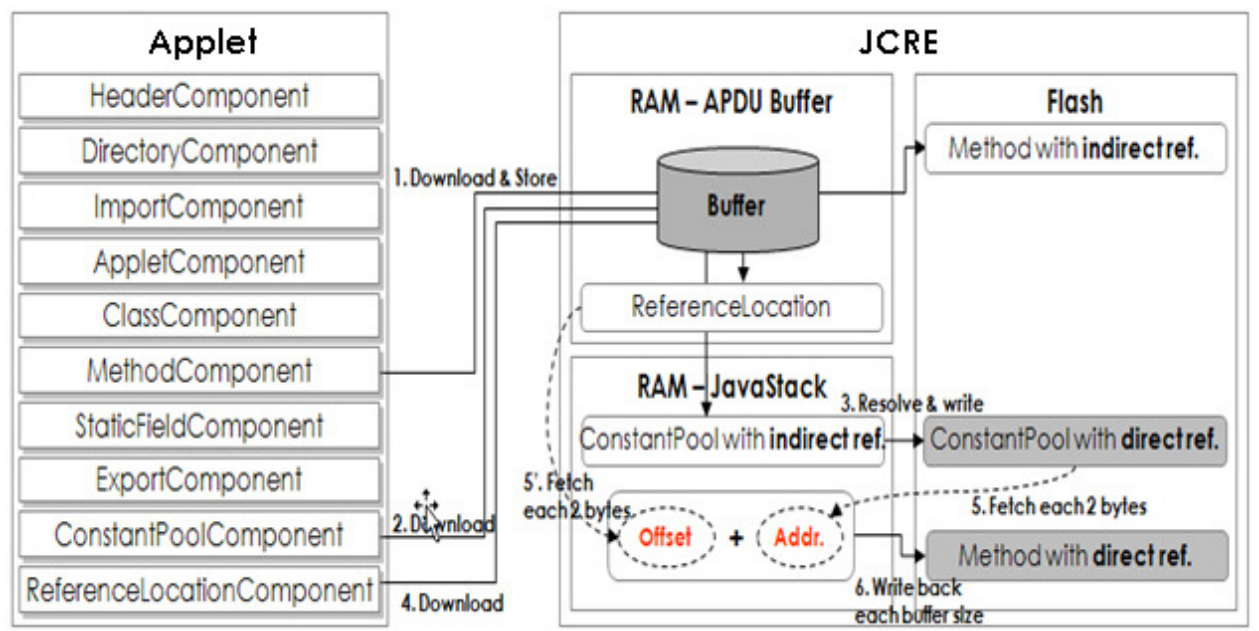

Fig. 1. The resolution and linking processes of the proposed efficient installation scheme. It uses available RAM space instead of Flash to resolve references for objects, static fields, variables and array. All data of component is transferred by a block that is 256 bytes.

identifies optimal block size suitable for reducing installation time. Fig. 1 shows our efficient scheme that is fully utilization of RAM space instead of Flash memory.

In order to evaluate the efficiency of the proposed installation scheme, we have conducted a series of experiments in the S3FS9QB, ARM 32 MCU smart card development environment. This single-chip CMOS micro-controller enabled development environment is specifically designed and packaged for Java Smart Card applications. The S3FS9QB has 8K bytes ROM for built-in boot loader, $256 \mathrm{~K}$ bytes program Flash, $8 \mathrm{~K}$ bytes RAM, and serial I/O interfaces [10].

\section{Result and discussions}

The installation process requires component downloading, resolution, and allocation. Hence the installation process should be evaluated in terms of communication time, between off-card and on-card, and of CPU processing time. In order to accurately estimate the CPU processing time of the on-card platform, we developed a new API that can be easily invoked to get CPU processing time by the Installer applet.

As we can see from Table I, the overall installation time required by the proposed our efficient scheme was significantly reduced as compared to the conventional scheme. Our scheme could reduce $38 \%$ of the communication time compared to the conventional scheme. The communication time reduction is more significant when the applet size is larger.

Table II shows the CPU processing time required for various applet installations. CPU processing time is mainly used for handling address resolutions. Using the RAM more efficiently during the resolution processes could out- 
Table I. Total installation time for the various applets (times in milliseconds).

\begin{tabular}{|l|c|c|c|c|}
\hline \multicolumn{1}{|c|}{ Applets } & Size(KB) & Conventional (37B) & Our scheme(256B) & Gain(\%) \\
\hline ChannelDemo & 3.15 & 132040 & 80721 & 39 \\
\hline JavaLoyalty & 2.04 & 127932 & 87222 & 32 \\
\hline JavaPurse & 10.70 & 394675 & 216987 & 45 \\
\hline ObjectDeletion & 5.76 & 285286 & 175727 & 38 \\
\hline PackageA & 3.77 & 167653 & 96114 & 43 \\
\hline PackageB & 2.13 & 134447 & 90834 & 32 \\
\hline PackageC & 0.68 & 61022 & 43488 & 29 \\
\hline Photocard & 2.32 & 118479 & 73785 & 38 \\
\hline RMIDemo & 1.75 & 107322 & 69051 & 36 \\
\hline Wallet & 2.05 & 99946 & 63096 & 37 \\
\hline EMV_Applet & 6.88 & 204041 & 112410 & 45 \\
\hline
\end{tabular}

Table II. Real CPU processing time for installation (times in milliseconds).

\begin{tabular}{|l|c|c|c|c|}
\hline Applets & Size(KB) & Conventional (37 B) & Our scheme(256 B) & Gain(\%) \\
\hline ChannelDemo & 3.15 & 44486.51 & 34131.47 & 23 \\
\hline JavaLoyalty & 2.04 & 41661.39 & 38754.86 & 7 \\
\hline JavaPurse & 10.70 & 147803.17 & 104525.17 & 29 \\
\hline ObjectDeletion & 5.76 & 108197.91 & 84969.29 & 21 \\
\hline PackageA & 3.77 & 62562.29 & 43403.18 & 31 \\
\hline PackageB & 2.13 & 45441.15 & 41548.25 & 9 \\
\hline PackageC & 0.68 & 15685.87 & 16504.98 & -5 \\
\hline Photocard & 2.32 & 40589.34 & 31009.97 & 24 \\
\hline RMIDemo & 1.75 & 36620.01 & 28862.75 & 21 \\
\hline Wallet & 2.05 & 31008.27 & 24950.73 & 20 \\
\hline EMV_Applet & 6.88 & 69037.32 & 50101.78 & 27 \\
\hline
\end{tabular}

perform the conventional Installation scheme. Address resolution is needed only for two components, namely, Method and ConstantPool components by using offset values of ReferenceLocation component. The CPU processing time depends on the file size of an applet, especially the size of Method and Constant Pool components. For example, BigApplet has the large file size and therefore has large Method and Constant Pool components. The CPU processing time for the BigApplet address resolution could be significantly reduced. However in some cases such as PackageC applet, which has smallest file size, the CPU processing time is slightly more than the processing time by the conventional scheme. The reason is that our scheme needs to parse components. The parsing time overhead for a small applet was slightly more than the performance gain obtained by effectively using the RAM for the resolution processes. Overall we could reduce about 20\% CPU processing time for applet installations by using our scheme.

We also tried to identify optimal block size that could maximally reduce installation time. As shown in Table III (a) and III (b), reducing communication overhead between off-card and on-card installer by the proposed scheme could be obtained when the block size is bigger than 165 bytes. The conventional method transfers block of size 37 bytes with acknowledgment between on-card and off-card.

Therefore the communication overhead is much bigger than that used in 
Table III. Applet installation time in terms of transferring block size (unit (\%)).

(a) Total applet installation times

\begin{tabular}{|l|c|c|c|c|c|c|c|}
\hline $\begin{array}{c}\text { Block size(B) } \\
\text { Applets }\end{array}$ & 69 & 101 & 133 & 165 & 197 & 229 & 256 \\
\hline ChannelDemo & 27 & 32 & 36 & 38 & 39 & 40 & 39 \\
\hline JavaLoyalty & 25 & 27 & 30 & 30 & 31 & 31 & 32 \\
\hline JavaPurse & 31 & 35 & 40 & 42 & 43 & 44 & 45 \\
\hline ObjectDeletion & 30 & 33 & 35 & 36 & 38 & 39 & 38 \\
\hline PackageA & 33 & 36 & 39 & 41 & 42 & 43 & 43 \\
\hline PackageB & 27 & 29 & 30 & 31 & 32 & 33 & 32 \\
\hline PackageC & 24 & 28 & 28 & 26 & 29 & 29 & 29 \\
\hline Photocard & 27 & 32 & 35 & 36 & 37 & 38 & 38 \\
\hline RMIDemo & 28 & 31 & 33 & 34 & 35 & 36 & 36 \\
\hline Wallet & 26 & 30 & 33 & 36 & 36 & 37 & 37 \\
\hline EMV_Applet & 25 & 34 & 39 & 42 & 44 & 45 & 45 \\
\hline Average & $\mathbf{2 8}$ & $\mathbf{3 4}$ & $\mathbf{3 5}$ & $\mathbf{3 6}$ & $\mathbf{3 8}$ & $\mathbf{3 8}$ & $\mathbf{3 8}$ \\
\hline
\end{tabular}

(b) CPU processing times

\begin{tabular}{|l|c|c|c|c|c|c|c|}
\hline $\begin{array}{c}\text { Block size(B) } \\
\text { Applets }\end{array}$ & 69 & 101 & 133 & 165 & 197 & 229 & 256 \\
\hline ChannelDemo & 9 & 15 & 20 & 22 & 23 & 24 & 23 \\
\hline JavaLoyalty & 0 & 1 & 4 & 5 & 6 & 6 & 7 \\
\hline JavaPurse & 14 & 20 & 24 & 26 & 27 & 28 & 29 \\
\hline ObjectDeletion & 10 & 15 & 17 & 19 & 21 & 22 & 21 \\
\hline PackageA & 15 & 24 & 26 & 28 & 30 & 31 & 31 \\
\hline PackageB & 2 & 4 & 5 & 6 & 8 & 9 & 9 \\
\hline PackageC & -9 & -7 & -6 & -10 & -5 & -5 & -5 \\
\hline Photocard & 11 & 17 & 20 & 21 & 22 & 23 & 24 \\
\hline RMIDemo & 13 & 16 & 18 & 19 & 21 & 21 & 21 \\
\hline Wallet & 7 & 10 & 14 & 18 & 18 & 20 & 20 \\
\hline EMV_Applet & 4 & 15 & 21 & 23 & 26 & 28 & 27 \\
\hline Average & $\mathbf{7}$ & $\mathbf{1 3}$ & $\mathbf{1 6}$ & $\mathbf{1 7}$ & $\mathbf{1 9}$ & $\mathbf{2 0}$ & $\mathbf{2 0}$ \\
\hline
\end{tabular}

the proposed efficient scheme. Our efficient scheme however has overhead for parsing components from the transferred data. The parsing overhead could be well offset by having block size bigger than 37 bytes. The peak performance could be obtained when the block size becomes larger than 165 bytes.

\section{Conclusion}

In this paper, we have investigated an efficient scheme for installing an applet efficiently in the Java Card platform. The salient features of our scheme are efficient installation of an application applet by fully utilizing available RAM space and by reducing communication overhead for downloading applet data with adjustable parameter tuning such as transferring data block size. The proposed novel installation scheme could reduce the installation time required for an applet by about $38 \%$ compared to the conventional installation scheme.

The Java Card 2.2.1 is designed with RAM size $1 \sim 2 \mathrm{~K}$ bytes. It is projected to have an on-card platform with the RAM size of about $6 \sim 10 \mathrm{~K}$ in near future. Our proposed scheme is therefore especially suitable and useful for improving the performance of the Java Card Platform by fully utilizing increased RAM space and asynchronous component transfer mode. 


\section{Acknowledgments}

The authors wish to thank Prof. Gwang Jung of Lehman college, City University of New York for helpful discussions and performing the experiments of the process. 